\title{
Two varieties of perceived length ${ }^{1}$
}

The perception of length depends on the mode of stimulation. When Ss were asked to judge the apparent lengths of blocks presented either visually or proprioceptively (between thumb and finger of one hand), visual length was proportional to block length, whereas proprioceptive length was an accelerating function of block length. When both visual and proprioceptive stimulation occurred simultaneously ( $S$ both saw and felt a block), the visual input was preemptive.

Although there is evidence that visual length is nearly proportional to stimulus length (e.g., Stevens \& Guirao, 1963; M. Teghtsoonian, 1965), lengths perceived by proprioceptive or kinesthetic means may be nonlinear with physical length and with each other. Many subjective magnitudes are nonlinearly related to stimulus magnitudes (e.g., Stevens \& Galanter, 1957). The possibility of differences among varieties of perceived length follows from the idea that led Berkeley (1950) to observe: "But if we take a close and accurate view of things, it must be acknowledged that we never see and feel one and the same object. That which is seen is one thing, and that which is felt is another .... the objects of sight and touch are two distinct things. It may perhaps require some thought to rightly conceive this distinction. And the difficulty seems not a little increased, because the combination of visible ideas hath constantly the same name as the combination of tangible ideas wherewith it is connected ...."

Jastrow (1886) gave experimental expression to Berkeley's observation when he presented lengths to $\mathrm{Ss}$ either visually (drawn lines), proprioceptively (block grasped between thumb and forefinger), or kinesthetically (slider moved between stops by arm), and required $\mathrm{Ss}$ to produce matching lengths either visually (by marking off a line), proprioceptively (by adjusting the width between thumb and forefinger), or kinesthetically (by moving the arm). All nine combinations of presentation and production were explored. He concluded that the perception of length depended on the mode both of experience and of expression.

Waterman (1917), in an approach similar to Jastrow's, had Ss match the size of circles laid on the palm of the hand and on the tip of the tongue by selecting circles from a visual display. "Tongue-size" and "palm-size" appeared to be related in different ways to visual size, although in both cases visual circles smaller than "touch" circles were required to make a match.

Recent investigations fall into three major groups. First, some have compared visual and tactile perceived lengths by means of the classical psychophysical methods of threshold measurement (Kelvin \& Mulik, 1958; Davidon \& Mather, 1966). Thus, a given visual extent may be chosen and a point of subjective equality determined for it on a scale of tactile length. These experiments have been restricted to one or two isolated values of physical length.

A second group of experiments involves artificial alterations of the relation between visual and proprioceptive-kinesthetic systems. Nielsen (1963) had S draw a straight line while watching a hand which $S$ thought to be his own but which in reality belonged to E's assistant; even when the seen hand deviated grossly from what S's own felt hand was doing, $S$ experienced the seen hand as belonging to himself. Hay, Pick, and Ikeda (1965) have discussed the phenomenon of "visual capture" in which the prismatically-altered visual system appears to capture the proprioceptive system: The felt position of the hand moves toward its seen position. Rock and Victor (1964) have shown that visual input predominates over touch when they are placed in conflict by optically altering the width of the visual stimuli. Harris (1965) has argued that the effects of inverting, displacing, or distorting the visual field may be explained most economically in terms of proprioceptive adaptation; he proposed that the visual system is stable and accurate while the proprioceptive system is relatively labile.

Thirdly, there are a number of experiments on the scaling of length with the direct psychophysical techniques described by Stevens (1958). Most of these deal with visual length, which has been shown to be linear with physical length (i.e., exponent approximately 1.0). (See Teghtsoonian \& Teghtsoonian, 1965, for a brief review.) Ronco (1963) presented lengths by having a blindfolded $S$ grasp a slider and move his arm until it touched a peg; mean magnitude estimations were approximately linear with physical length. Teghtsoonian and Teghtsoonian (1965) scaled seen and felt lengths of metal rods. Magnitude estimations of apparent length were linearly related to physical length both when the rods were only seen or only felt with the two index fingers. Within the range explored, the two ways of apprehending length were interchangeable. Stanley (1966) confirmed the finding for proprioceptive length of rods (exponent 0.94 ) and extended it to index-finger separation without rods (exponent 1.05) Gogel, Wist, and Harker (1963) had S make kinesthetic adjustments of the separation of an unseen pair of rods to match the separation of another pair of rods presented visually; the kinesthetic matches appeared proportional to the physical length of the visual interval. On the assumption that visual length is proportional to physical length, they concluded that kinesthetic length is also.

Stevens and Stone (1959) scaled the subjective widths of blocks placed between thumb and finger of one hand, using the

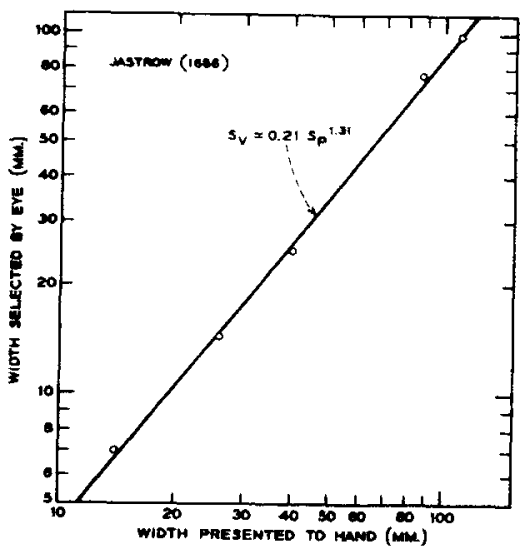

Fig. 1. Visually-selected width $\left(S_{v}\right)$ required to match proprioceptively. presented width $\left(S_{p}\right)$. Unseen blocks grasped between thumb and forefinger were matched by marking off a portion of a seen line, or by selecting one of an array of seen lines. The data are from Jastrow (1886), and the points were estimated from a figure. This function and several others are said to represent "the average of experiments upon from three to seven individuals and the result of about 1,800 observations." 


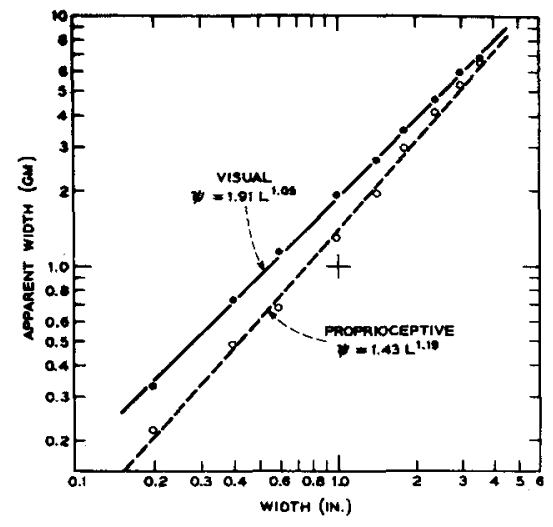

Fig. 2. Apparent width of blocks presented visually and proprioceptively. Fifteen Ss judged with visual presentation on one occasion (filled circles) and with proprioceptive, finger-span, presentation on another (open circles). The + shows the point ( 1 in., apparent width 1 ).

method of magnitude estimation, and obtained a power function for finger span with an exponent of 1.33. This appears to be the first modern study reporting a scale of length that is markedly nonlinear. It is in close agreement with Jastrow's earlier measurements of visual widths selected to match finger spans. On the assumption that visual width is linearly related to physical width, Jastrow's data can be fitted by a power function with an exponent of 1.31, as shown in Fig. 1. Mashhour and Hosman (1968) obtained a power function for finger span with an exponent of 1.26 when the middle value of the test series of 11 widths was used as the standard in a magnitude-estimation procedure.

In our first experiment, Ss made magnitude estimations of a set of block widths presented either visually or to be spanned by thumb and finger. In a second experiment, Ss made magnitude estimations of these same stimuli when they were incorporated with a visual frame, an analog to the "frame" effected by maximum thumb-and-finger span. In the third experiment, Ss judged the widths of blocks seen and felt concurrently.

\section{EXPERIMENT 1:}

\section{VISUAL VS PROPRIOCEPTIVE} PRESENTATION

\section{Method}

Stimuli. The stimuli were nine wooden blocks, 6 in. long and $0.5 \mathrm{in}$. high; their widths were $0.20,0.39,0.59,0.98,1.38$, $1.77,2.36,2.95$, and 3.54 in. They were cut from hard wood and milled to the desired widths (which were multiples of $5 \mathrm{~mm}$ ).

Subjects. Ss were 15 women undergraduates.
Procedure. Each S was run at two separate sessions, 1 week apart. Eight Ss were given visual presentation in the first session and proprioceptive in the second; the order was reversed for the remaining seven. Ss were alternately assigned to one or the other order as they appeared. During the first session, the instructions were read to $S$ : She was to assign numbers to the blocks to represent how wide they looked (or felt) to her. She could assign any number that seemed appropriate to the width of the first block, and her other judgments should be proportional to the apparent width of the other blocks. (If $S$ asked whether she should make her judgments in inches, she was told that she could if she wished, but that she need not.) Then the nine blocks were presented in two successive random orders; different random orders were used for each $S$. At the second session, the same procedure was repeated, with variations appropriate to the modality.

For visual presentation, the blocks were placed one at a time on a black-topped table in front of $S$, with their variable dimension parallel to the front of the table. For proprioceptive presentation, $S$ was blindfolded. She rested her elbow on the table top, and $E$ placed a block on her thumb; $S$ lowered her middle finger until she was grasping the block firmly between thumb and finger, supporting its weight herself. When $S$ made her judgment, $E$ removed the block and placed a new one.

\section{Results}

A straight line was fitted to the geometric means in log-log coordinates by the method of least squares. Figure 2 shows the results for visual judgments (filled circles) and for proprioceptive judgments (open circles). For visual and for proprioceptive apparent length, respectively, only $0.1 \%$ and $0.3 \%$ of the variance in log mean judgments was not accounted for by the linear regression; the goodness of fit indicated that apparent length was a power function of physical length. Visual apparent length gave an exponent of $1.046 \pm 0.013$, while thumb-and-finger apparent length gave an exponent of $1.193 \pm 0.028$. (The error terms are estimated standard errors.)

In order to obtain scores appropriate to a statistical evaluation of the effects of presentation mode and of period of testing, power functions were fitted by least squares for each $\mathrm{S}$. The obtained exponents of individual power functions were subjected to an analysis of variance. ${ }^{3}$ Only the effect due to sensory mode-visual vs proprioceptive-was significant $(.01<\mathrm{p}<.05)$. Neither period of testing (first vs second) nor the interaction of mode with period was significant $(p>.10)$.
The means of the two distributions of individual exponents were, of course, the same as the mean exponents shown in Fig. 2. The standard deviations were 0.073 for visual presentation and 0.150 for proprioceptive presentation.

Finally, the variability of the $l o g$ judgments themselves was found to be approximately the same for the two situations, and nearly constant (in log units) over the range of stimuli employed. For visual length, the standard deviation of $\log$ magnitude estimations of a given stimulus ranged from 6.3 to 6.5 decilogs. For proprioceptive length, the corresponding values were 5.8 to 6.4 decilogs. These measures of variability were calculated for logarithms of the judgments as given by $\mathrm{Ss}$, without equalizing moduli.

\section{Discussion}

Judgments of visual length were different from judgments of proprioceptive length in this situation: Doubling the block width approximately doubled its visual width but increased its felt width by a factor of 2.3. The nearly linear relation of visual and physical length is well documented, and these results provided no exception to the rule of proportionality. The nonlinearity of proprioceptive length with physical length confirms the results of earlier studies (Jastrow, 1886; Stevens \& Stone, 1959; Mashhour \& Hosman, 1968) and demonstrates that the effect in those studies did not depend upon the shortened range of values necessitated by finger-span presentation, since the same range did not alter the linearity between visual and physical length.

Although the several studies agree that the proprioceptive length function is accelerated, the data of Stevens and Stone (1959), Mashhour and Hosman (for one condition) (1968), and Jastrow (1886) yield exponents of 1.3 , higher than ours of 1.2. Stevens and Stone (1959) pointed out that judgments of narrow stimuli were too high: Their functions on $\log -\log$ coordinates showed upward curvature at the low end. In fitting lines by eye, they therefore gave less weight to points at smaller stimulus values. Neither Jastrow (1886) nor Mashhour and Hosman (1968) used stimuli smaller than $10 \mathrm{~mm}$, and their functions exhibited little upward curvature at the low end. Our data were fitted by the method of least squares, which gives equal weight to all points, including those for 5 and $10 \mathrm{~mm}$; a line fitted by least-squares omitting these points gave an exponent of 1.3. It appears that a power function with exponent 1.3 characterizes magnitude estimations of finger span for widths greater than $5.10 \mathrm{~mm}$; as widths become narrower, Ss are unable to perform the matching operation requested by $\mathrm{E}$. 
Exponents in magnitude estimation experiments are typically lower than those in magnitude production experiments, and the magnitude of this regression effect varies across continua (Stevens \& Greenbaum, 1966). The regression effect for visual length has been shown to be very small (Stevens \& Guirao, 1963), whereas a sizeable regression effect for proprioceptive length has been demonstrated by Stevens (1969) using data from Mashhour and Hosman (1968). Stevens estimated the production exponent to be 1.9 . We might therefore expect the difference between magnitude-production exponents for visual and proprioceptive length to be somewhat greater than the difference we obtained between magnitude-estimation exponents.

The difference between the scale factors of the two power functions which is evident in Fig. 2 turned out to be attributable to only three $\mathrm{Ss}$, who altered their range of numbers from session to session; 12 Ss who used approximately the same range of numbers in both sessions had an average scale factor of 1.0 for both visual and proprioceptive length. Evidently many Ss were influenced in their choice of range by the learned scale of inches: $50 \%$ assigned a number between 0.875 and 2.50 to the 0.98 -in. block presented visually.

One objection to the conclusion that a difference exists between the two scales of length is that, although the physical stimuli, the blocks, are identical in both presentation conditions, there may not be phenomenal equality between the two conditions. The thumb and finger can open to a maximum span of about 6 or 7 in. when $S$ is feeling the blocks, while the seeing $S$ might reasonably expect a much wider stimulus; thus there is a ceiling on proprioceptive presentation which is both more obvious and much lower than the ceiling on visual presentation. Since we have previously found an exponent of 1.0 for index finger separation, it could be that the "basic" exponent for thumb-and-finger separation is 1.0, yet in Experiment 1 it is elevated by factors extraneous to, but confounded with, the mode of presentation. In the next experiment, the blocks were seen through an opening $6 \times 1 / 2$ in., thus providing a ceiling for visual block size like that which occurred for proprioceptive block size.

\section{EXPERIMENT 2:}

\section{Method}

\section{PROVIDING A VISUAL FRAME}

Stimuli. The stimuli were those used in Experiment 1.

Subjects. Ss were 15 women undergraduates.

Procedure. Only one session was run. The standard instructions were used; $S$ was to assign numbers to represent how the lengths of the blocks looked to her. There was no identified standard or assigned modulus. The nine blocks were presented in two successive random orders, different for each $S$.

S sat on a stool 34 in. high, looking down on the display, which was placed on a desk top $30 \mathrm{in}$. high. What she saw was a black background, $22 \times 30$ in., with a centrally-located white slot, $6 \times 1 / 2$ in. (the long dimension oriented vertically with respect to $S$ ). The wooden blocks appeared in this slot; thus they were apparently $1 / 2$ in. across, and of variable height, with a possible maximum of $6 \mathrm{in}$. The unfilled white space was always at the top of the slot.

\section{Results and Discussion}

Geometric means were determined for all judgments at each stimulus and plotted against block width in logarithmic coordinates. The result, shown in Fig. 3, is well fitted by the power function $\psi=5.23 \mathrm{~L}^{0.95}$. Apparently, a visual frame does not cause visual judgments to resemble the proprioceptive judgments of Experiment 1. The exponent is typical of those obtained in judgments of visual length, in spite of the ceiling imposed on stimulus width and in spite of the narrowing of the constant dimension of the blocks from 6 to 0.5 in.

The conclusion of Experiment 1, that visual and proprioceptive length are different in some respects, receives at least indirect support from the outcome of Experiment 2. The difference, at any rate, is not attributable to different expectations regarding stimulus values. We are led then to the case in which $S$ has both visual and proprioceptive information about the same object simultaneously. This includes the everyday situation in which we pick up something and look at it.

\section{EXPERIMENT 3:}

\section{CONCURRENT VISUAL AND}

\section{PROPRIOCEPTIVE STIMULATION}

\section{Method}

Stimuli. The nine blocks from Experiment 1 were used.

Subjects. Sixteen women undergraduates were Ss.

Procedure. Only one session was run. The standard instructions were used: $S$ was to assign numbers to represent the widths of the blocks. No standard was identified and no modulus assigned. No reference was made to a specific modality-neither "looked" nor "felt" was used-but S was to respond in terms of how wide the block "seemed" to her. The method of presentation was the same as that for proprioceptive presentation in

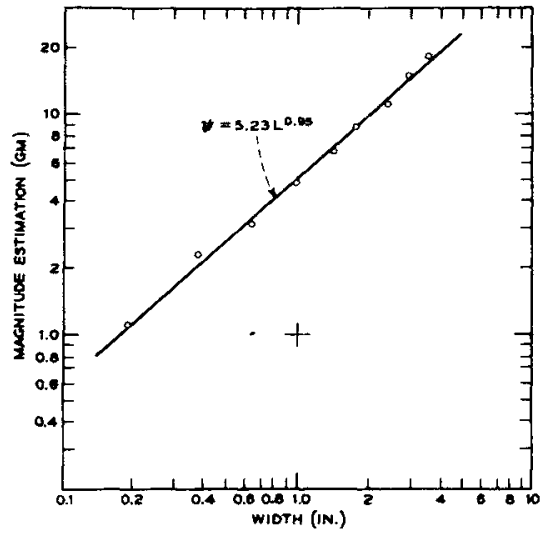

Fig. 3. Apparent width of blocks presented within a visual frame $6 \times 0.5$ in. $N=15$. The + shows the point ( 1 in., apparent width 1 ).

Experiment 1, with the block placed by $\mathrm{E}$ between S's thumb and finger, except that $\mathrm{S}$ was not blindfolded, and was free to look at the block at the same time she felt it. In other respects, the procedure was the same as that of Experiment 1 for the first session.

\section{Results and Discussion}

All judgments were transformed to logarithms; each S's two judgments for a given stimulus were then averaged to give a single score. These scores were subjected to a specially constructed analysis of variance. ${ }^{4}$ This analysis indicated that $\mathrm{Ss}$ differed significantly among themselves with respect to both intercepts $(p<.01)$ and slopes $(p<.01)$ of individual least-square fits. The effect of stimuli was significant $(p<.01)$; but the deviation from mean linear regression was not $(p>.10)$, and $99.9 \%$ of the variance was accounted for by the linear regression. Figure 4 shows geometric means; the fitted line has a slope of $1.036 \pm 0.096$ (the standard error of the estimate obtained from variance components of the analysis of variance).

The exponent from this experiment, 1.04 , was very close to that obtained in Experiment 1, 1.05, for visual length without visual frame, but greater than that obtained in Experiment 2;0.95, for visual length with visual frame. A single-factor analysis of variance on individual exponents from the three experiments showed a significant $\mathrm{F}(.01<\mathrm{p}<.05)$; a Newman-Keuls test confirmed that visual length with visual frame was different $(.01<\mathrm{p}<.05)$ from the other two conditions, which were not different from each other $(p>.10)$. The effect of visual frame in Experiment 2 was unexpected, and we cannot explain it. The similarity of exponents for visual length from 


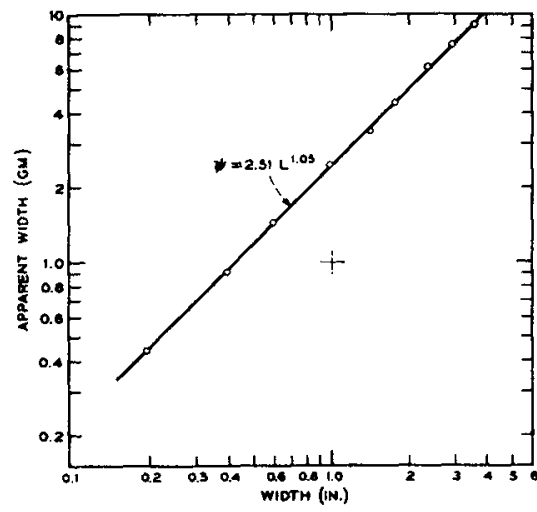

Fig. 4. Apparent width of blocks with concurrent visual and proprioceptive (finger-span) presentation. $N=15$. The + shows the point ( 1 in., apparent width 1$)$.

Experiments 1 and 3 suggests that $S_{s}$ in Experiment 3, although receiving both visual and proprioceptive information, were giving judgments characteristic of visual information alone.

The outcome of this experiment is consistent with the visual capture phenomenon reported by Hay, Pick, and Ikeda (1965) and the visual dominance phenomenon reported by Rock and Victor (1964). In all, the visual system predominates. In this experiment, the conflict between sight and touch was a "natural" one, requiring no distortions or displacements introduced by $E$, and the "natural" way of responding was one of reliance on visual input.

\section{SUMMARY AND CONCLUSIONS}

When Ss were asked to judge the apparent lengths of blocks presented either visually or proprioceptively-between thumb and finger of one hand-apparent length was related to physical length by a power function. The exponent depended upon the presentation modality: Visual length grew as approximately the first power of physical length, but thumb-and-finger length grew as the 1.2 power. These differences did not depend on the presence of the proprioceptive "frame" provided by limited finger span, and they are consistent with the work of other investigators. Length, while apprehended both by sight and by touch, does not seem to be invariant with mode of presentation. (The fact that proprioceptive length grows with an exponent near 1.0 if $S$ puts one index finger on each end of a rod, or simply holds the two fingers a given distance apart, but with exponent in the range 1.2 to 1.3 if he holds a block between thumb and finger of one hand, suggests that proprioceptive length itself is not characterized by a single scale.)

When both visual and proprioceptive stimulation occurred simultaneously, the visual input was preemptive. In a natural judging situation, when the two modes of presentation would produce different length judgments, Ss gave answers characteristic of the visual mode.

\section{REFERENCES}

BERKELEY, G. A new theory of vision New York: Dutton, 1950.

DAVIDON, R. S., \& MATHER, J. H Cross-modal judgments of length. American Journal of Psychology, 1966, 79, 409-418.

GOGEL, W. C., WIST, E. R., \& HAR KER, G. S. $A$ test of the invariance of the ratio of perceived size to perceived distance. American Journal of Psychology, 1963, 76, 537-553.

HARRIS, C. S. Pexceptual adaptation to inverted, reversed, and displaced vision. Psychological Review, 1965, 72, 419-444.

HAY, J. C., PICK, H. L., JR., \& IKEDA, K Visual capture produced by prism spectacles. Psychonomic Science, 1965, 2, 215-216.

JASTROW, J. The perception of space by disparate senses. Mind, 1886, 11, 539-554.

KELVIN, R. P., \& MULIK, A. Discrimination of length by sight and touch. Quarterly Journal of Experimental Psychology, 1958, 10, 187-192.

MASHHOUR, M., \& HOSMAN, J. On the new "psychophysical law": A validation study. Perception \& Psychophysics, 1968, 3, 367-375.

NIELSEN, T. I. Volition: A new experimental approach. Scandinavian Journal of Psychology, $1963,4,225-230$.

ROCK, I., \& VICTOR, J. Vision and touch: An experimentally created conflict between the two senses. Science, 1964, 143, 594-596.

RONCO, P. G. An experimental quantification of kinesthetic sensation: Extent of arm movement. Journal of Psychology, 1963, 55, 227-238.

STANLEY, G. Haptic and kinesthetic estimates of length. Psychonomic Science, 1966, 5, 377-378.
STERNBERG, S. High-speed scanning in human memory. Science, 1966, 153, 652-654.

STEVENS, S. S. Problems and methods of psychophysics. Psychological Bulletin, 1958, 54, 177-196.

STEVENS, S. S. On predicting exponents for cross-modality matches. Perception \& Psychophysics, 1969, 6, 251-256.

STEVENS, S. S., \& GALANTER, E. H. Ratio and category scales for a dozen perceptual continua. Journal of Experimental Psychology $1957,54,377-411$.

STEVENS, S. S., \& GREENBAUM, H. B. Regression effect in psychophysical judgments. Perception \& Psychophysics, 1966, 1, 439-446.

STEVENS, S. S., \& GUIRAO, M. Subjective scaling of length and area and the matching of length to loudness and brightness. Journal of Experimental Psychology, 1963, 66, 177-186.

STEVENS, S. S., \& STONE, G. Finger span: Ratio scale, category scale, and jnd scale. Journal of Experimental Psychology, 1959, $57,91-95$.

TEGHTSOONIAN, M. The judgment of size. American Joumal of Psychology, 1965, 78, $392-402$.

TEGHTSOONIAN, M., \& TEGHTSOONIAN, R. Seen and felt length. Psychonomic Science, $1965,3,465-466$.

WATERMAN, C. N., JR. Hand-tongue space perception. Journal of Experimental Psychology, 1917, 2, 289-294.

WINER, B. J. Statistical principles in experimental design. New York: McGraw-Hill, 1962.

NOTES

1. This research was supported by USPHS Grant MH 11810-01; the experiments were done at Smith College. We thank Sandra Eber, who ran $S s$ and assisted in analysis of data. The preparation of this manuscript was carried out while we were visitors in the Behavioral Research Laboratory of the Bell Telephone Laboratories, Inc., Murray Hill, N.J., on leave of absence from Smith College. We are grateful to Saul Sternberg and Charles Harris for their helpful discussions and criticisms of the manuscript. $A$ version of this paper was read at the meetings of the Psychonomic Society, St. Louis, 1966.

2. Address: Department of Psychology, Smith College, Northampton, Massachusetts 01060

3. The model is described by Winer (1962) for a Latin square design with repeated measures on one factor. The group effect is considered the Mode by Period interaction; it is tested against $S$ s within groups. Scores for the missing $S$ were estimated from the appropriate group means.

4. We are indebted to S. Stemberg for this technique, which he developed for application to a formally similar problem (Sternberg, 1966).

(Accepted for publication March 23, 1970) 